\title{
Concentración de sectores intensivos en conocimiento y de alta tecnología: el caso de España
}

\author{
Dr. Juan Vicente García Manjón (I)
}

\begin{abstract}
The Knowledge society is based on the growing importance of the service sector in the whole economy. Knowledge intensity and technology intensity variables make up a range of services showing differential characteristics such as the geographic concentration level of these sectors. In the present article we analyse the concentration trends in knowledge and high technology intensive sectors, taking into account variables such as the number of companies, employment and wages and salaries.

Keywords: Knowledge economy, knowledge intensity, technology intensity,

high technology, concentration.

\section{Resumen}

La sociedad del conocimiento se basa en la creciente importancia del sector servicios. Las variables de intensidad de conocimiento e intensidad tecnológica conforman una serie de servicios que presentan unas características diferenciales, como es el grado de concentración de estos sectores a nivel geográfico. En el presente artículo se analizan las tendencias de concentración en España en sectores intensivos en conocimiento de alta tecnología, teniendo en cuenta las variables de número de empresas, número de ocupados y salarios.
\end{abstract}

Palabras clave: Economía del conocimiento, intensidad de conocimiento, intensidad tecnológica, alta tecnología, concentración.

(I) Facultad de Ciencias Jurídicas y Económicas. Universidad Europea Miguel de Cervantes. Calle Padre Julio Chevalier, $\mathrm{n}^{\circ} 2 . V^{2}$ alladolid (470I2). España. Tel. + 34.983. 228508 (ext. 236). E mail jvgarciam@uemc.es 


\section{Introducción}

Los sectores intensivos en conocimiento de alta tecnología están conformados por el sector de las comunicaciones, el de investigación y desarrollo y el de informática. Estos sectores incorporan dos características diferenciales respecto a otros sectores, como son la combinación de la intensidad de conocimiento y la intensidad tecnológica y son claves para el desarrollo de la denominada sociedad del conocimiento. De acuerdo con Sundbo (2002), estos sectores tienen un alto valor en la economía del conocimiento. Dentro de estos servicios, son los de base científica y tecnológica los que más valor añadido aplican dentro de su proceso de prestación, siendo considerado el conocimiento como la principal materia prima de este tipo de empresa.

En concreto, el gasto realizado en I+D por parte de las empresas relacionadas con estos servicios avanzados, es proporcionalmente mayor que el peso que estas tienen en la economía y el desarrollo de los servicios intensivos en conocimiento en particular y suponen un detonante para la mejora de la competitividad de la economía en general.

Los servicios intensivos en conocimiento de alta tecnología generan empleo de alto valor añadido, especializado, con contratación de personal con educación superior, el cual sigue requiriendo inversión en su posterior capacitación. Estos sectores también inducen procesos de innovación en sus clientes a través de la realización de trabajos y proyectos a medida en sus organizaciones.

Según autores como Vence y Rodil (2003), Mas (1992), Martinelli, F (2002) o Rubalcaba (1998), estos sectores se encuentran altamente concentrados principalmente en las zonas con gran peso industrial y en muchos casos ligados a las grandes capitales.

Son habituales los estudios que han analizado las tendencias de concentración de los sectores intensivos en conocimiento a nivel europeo, pero muchos menos los que han abordado el análisis para entornos nacionales, principalmente debido a la menor disponibilidad de datos con desagregación a nivel regional o local. Sin embargo, su estudio a estos niveles se torna especialmente interesante debido a la relevancia que los mismos tienen para las economías regionales.

Es por ello que se plantea esta investigación para el estudio del caso de España, en el que se aborda el análisis del sector de servicios intensivos en conocimiento de alta tecnología con el objetivo de aportar luz sobre las pautas de concentración geográfica que se producen. La investigación se muestra perti- nente y puede tener aplicación en el diseño de políticas públicas de carácter regional para la promoción del desarrollo de este tipo de sectores.

\section{Marco teórico}

\section{La economía del conocimiento: intensidad de conoci- miento e intensidad tecnológica.}

Pese a la aparente novedad del término sociedad del conocimiento, este fue acuñado por Drucker, P (1969) en su obra "La era de la discontinuidad". Drucker se basa en trabajos anteriores como el de Fritz Machlup que a través de su obra "La producción y distribución de conocimiento en los Estados Unidos" popularizó el concepto de sociedad de la información. Posteriormente en los años 80 , estos conceptos fueron difundidos de la mano de autores como Daniel Bell, John Naisbitt y Alvin Toffler.

La sociedad del conocimiento toma como base la denominada sociedad de la información y se nutre de un nuevo recurso productivo que es el capital humano, el cual se caracteriza por su capacidad de generar valor. La sociedad del conocimiento engloba aspectos tales como la globalización, el desarrollo tecnológico, la actualización de habilidades, las organizaciones en red,...teniendo todos ellos un gran impacto en el mundo económico.

Una economía basada en el conocimiento es una economía en la cual la creación, distribución y uso del conocimiento es el mayor propulsor de crecimiento, riqueza y empleo Heng et al. (2002), siendo una de sus características la velocidad acelerada a la que ese conocimiento se crea, se acumula y seguramente se deprecia David y Foray (2002).

Una de las singularidades de las economías basadas en el conocimiento es el creciente peso del sector terciario. Las economías modernas se están convirtiendo en economías de servicios desde hace décadas, habiendo pasado cualquier punto de retorno a sistemas anteriores, Fuchs (1968). Dentro de la estructura económico-productiva a nivel mundial, el sector servicios muestra su vigor tanto por su importancia en las ocupaciones como en su aportación al VAB (Valor Añadido Bruto) y a la creación de nuevas empresas. El sector servicios, con roles dispares a nivel económico, representa dos tercios del empleo en las economías europeas e incluye los sectores más dinámicos en términos de crecimiento de empleo y de la productividad en las últimas décadas.

Para comprender el papel clave que los servicios juegan en la economía del conocimiento, hemos de empezar analizando las 
diferencias entre productos (bienes tangibles) y servicios. Así, según Vermeulen (200I) la primera diferencia se basa en la intangibilidad de los servicios. La producción de los servicios coincide con el momento en el que estos se consumen y esto implica que no pueden ser almacenados. Además los servicios tienen un carácter heterogéneo pese a la posibilidad de estandarización.

Esa heterogeneidad en los servicios a la que hacíamos referencia atiende a las características diferenciales de su propia naturaleza, siendo una de estas la intensidad de conocimiento inherente a cada tipología de servicios. La intensidad de conocimiento está centrada en el distinto grado de conocimiento que requiere la prestación de un servicio en cuestión. Autores como Hauknes (1999) asumían la dificultad que existe en delimitar lo que entendemos por intensidad de conocimiento y medirlo, debido a que la intensidad de conocimiento refleja la integración de ciencia o tecnología con un producto genérico o específico y puede ser definido como una combinación de conocimiento incorporado en nuevo equipamiento, personal o intensidad de I+D.

Otra forma de acercarse al concepto de intensidad de conocimiento, según el mismo autor, es afrontarlo desde el punto de vista del conocimiento necesario por parte del proveedor y del consumidor. Siguiendo esta aproximación, podemos decir que los servicios técnicos, el software a medida, las telecomunicaciones y los servicios de investigación y desarrollo estarían en el cuadrante de mayor intensidad de conocimiento, constituyendo los servicios de carácter más avanzado.

El papel de los servicios intensivos en conocimiento' resulta especialmente relevante en el conjunto de la economía. En este sentido el Consejo de Lisboa de la Unión Europea del año 2000 señaló la importancia de los servicios intensivos en conocimiento por su potencial de crecimiento económico y su papel como generador de empleo. De hecho, los KIS pueden ser considerados como una de los subsectores más relevantes de la economía basada en el conocimiento Muller y Zenker (200l) y son considerados como el componente más dinámico del sector servicios en los países industrializados Strambach (200I). En esta línea se expresan autores como Miles et al. (1995) y Abramovitz y David (1996) quienes defienden la identificación de los servicios de valor añadido con aquellos que son intensivos en conocimiento.

Sin embargo la intensidad de conocimiento no es el único parámetro a considerar si queremos estudiar qué características diferenciales presentan los servicios de valor añadido frente a otros que no lo son. En este sentido la intensidad tecnológica también se presenta como un variable relevante a considerar en nuestro análisis.

La intensidad tecnológica se mide en relación al uso que los servicios hacen de la tecnología, al propio servicio como origen de la tecnología y a los servicios como agentes de transferencia de la propia tecnología, Hauknes (1999).

Según Esteban (2006) un servicio intensivo en tecnología es "una actividad de naturaleza intangible que normalmente se genera por la interacción entre un cliente y una organización proveedora, pública o privada, en un mercado industrial, con el objeto de obtener una solución basada en conocimiento científico y/o tecnológico para el problema del cliente".

La dicotomía entre intensidad de conocimiento e intensidad tecnológica ha dado lugar a la categorización de los servicios atendiendo a las dos variables anteriores. La cuestión ha sido tratada desde el sistema estadístico europeo (EUROSTAT) en el que se aborda una clasificación de los servicios atendiendo a la intensidad de conocimiento e intensidad tecnológica. De esta manera EUROSTAT distingue entre servicios intensivos en conocimiento y servicios menos intensivos en conocimiento.A su vez para aquellos servicios calificados como intensivos en conocimiento, EUROSTAT realiza una clasificación según el detalle de la Tabla I que aparece a continuación.

I Knowledge Intensive Services (KIS) en terminología en inglés. 
Tabla I

Clasificación de los servicios intensivos en conocimiento. Fuente: EUROSTAT

\begin{tabular}{lll}
\hline \multicolumn{3}{c}{ Clasificación de los servicios intensivos en conocimiento } \\
\hline $\begin{array}{l}\text { Servicios intensivos en } \\
\text { conocimiento de alta tecnología }\end{array}$ & $(64)$ & Correos y telecomunicaciones \\
& $(73)$ & Informática y actividades conexas \\
\hline $\begin{array}{l}\text { Servicios de mercado intensivos y desarrollo } \\
\text { en conocimiento }\end{array}$ & $(61)$ & Transporte marítimo \\
& $(62)$ & Transporte aéreo \\
& $(70)$ & Actividades inmobiliarias \\
& $(71)$ & Alquiler de maquinaria \\
& $(74)$ & Otros servicios a empresas \\
\hline $\begin{array}{l}\text { Servicios financieros intensivos } \\
\text { en conocimiento }\end{array}$ & $(65)$ & Intermediación financiera \\
& $(66)$ & Seguros y fondos de pensiones \\
conocimiento & $(67)$ & Actividades auxiliares a la intermediación financiera \\
\hline
\end{tabular}

Como podemos ver en la tabla anterior, los servicios intensivos en conocimiento se componen de servicios intensivos en conocimiento de alta tecnología, servicios de mercado intensivos en conocimiento, servicios financieros intensivos en conocimiento y otros servicios intensivos en conocimiento.

Interés especial merecen los denominados servicios intensivos en conocimiento de alta tecnología (KIS_HT) ya que en ellos coinciden las dos variables consideradas en nuestro análisis, la de intensidad de conocimiento y la de intensidad tecnológica. En este sector se encuentran los correos y telecomunicaciones, la informática y actividades anexas y la investigación y desarrollo.

\section{Concentración geográfica de los servicios intensivos en conocimiento}

Normalmente los servicios intensivos en conocimiento y en particular aquellos intensivos en tecnología suelen estar altamente concentrados en determinadas áreas a nivel nacional o europeo. Así está refrendado por los estudios sobre la investigación universitaria y la I+D en general, como componentes claves de la producción de conocimiento, evidenciándose una concentración geográfica, y de forma muy especial en las regiones metropolitanas, Vence y Rodil (2003).

Otros autores como Mas (1992) ya apuntaron que los servicios intensivos en conocimiento no se distribuían de forma homogénea a nivel geográfico, sino que esa clase de actividades tendían a concentrarse en las grandes ciudades y en particular en las capitales. Esta concentración puede ser debida a causas como la necesidad de la cercanía a los clientes y que al realizarse esta en grandes aglomeraciones industriales se consiga de esta manera las consiguientes economías de escala.

También autores como Vence (2007) defiende que los servicios intensivos en conocimiento están caracterizados por la asimetría territorial. Este autor afirma que los patrones de especialización productiva regional en los distintos servicios intensivos en conocimiento presentan una asimetría importante en la importancia relativa de cada uno de los sectores considerados. La tendencia a la concentración de los servicios intensivos en conocimiento se da en mayor medida según Vence en los servicios intensivos en conocimiento de alta tecnología de 
forma muy importante y de forma más moderada en los servicios financieros, de mercado y otros servicios.

Otros autores como Rubalcaba (1998) han estudiado las causas de esta concentración. Entre los factores analizados están los del volumen de la demanda, el grado de internacionalización de los focos de producción y de los clientes y el grado de standardización o especialización de la prestación del servicio.

Martinelli (2002) apunta que en lo que se refiere a regiones periféricas o menos desarrolladas, el desarrollo de los servicios intensivos en conocimiento encuentra barreras a su desarrollo, ya que aunque al final surja la demanda de este tipo de servicios en estas regiones no necesariamente activa la oferta local. De la misma manera afirma que en realidad puede hacer crecer a proveedores especializados en otras regiones. Por otra parte, el mismo autor defiende que las regiones periféricas cuentan con la barrera de la lejanía de estas con los grandes centros de demanda, y teniendo en cuenta que este tipo de servicios se presta en relación con el cliente, esto supone una merma de las posibilidades de que este elija proveedores alejados de la sede de la compañía.

\section{Objetivos, metodología y datos utilizados}

\section{Objetivos de la investigación}

A través del presente artículo de investigación presentamos el análisis de las tendencias de concentración de servicios intensivos en conocimiento de alta tecnología ${ }^{2}$ (KIS_HT) para el caso de España. El análisis se muestra pertinente con el objetivo de aportar luz sobre el comportamiento del sector de servicios intensivos en conocimiento de alta tecnología debido a la relevancia que tienen estos sectores dentro de la denominada economía del conocimiento y en tanto generadores de innovación en el resto de sectores industriales y de servicios. Además en estos sectores confluyen las cualidades de intensidad de conocimiento e intensidad tecnológica lo que les confiere características diferenciales respecto a los sectores intensivos en conocimiento y sectores de alta tecnología in- dustrial.

\section{Metodología y datos utilizados}

La metodología utilizada tiene como objetivo el de investigar las tendencias de concentración del sector KIS_HT en España y los subsectores que lo componen. Para ello, se toma como unidad de análisis la comunidad autónoma (NUT $2^{3}$ ) y como población las diecisiete comunidades autónomas españolas. El sector KIS_HT, como aparece referenciado con anterioridad, está compuesto por los sectores de informática (CNAE 72), comunicaciones (CNAE 64) e investigación y desarrollo (CNAE 73).

A la hora de afrontar un análisis de este tipo nos encontramos con un primer reto que es la disponibilidad de datos del sector KIS_HT y en especial de datos sectoriales a nivel regional en España. En este sentido se han manejado principalmente fuentes a nivel nacional, DIRCE ${ }^{4}$, y a nivel europeo (EUROSTAT ${ }^{5}$ ). Los datos disponibles nos han permitido analizar las variables de número de empresas, número de ocupados y salarios para el sector y subsectores de referencia.

Para cada una de las variables de referencia se ha realizado un análisis descriptivo en la mayoría de los casos, incluyendo análisis de evolución temporal cuando la existencia de datos lo ha hecho posible.

Por otra parte, para abordar el objetivo principal de la investigación, se han llevado a cabo análisis de concentración recurriendo a uno de los índices más utilizados en la literatura científica, el índice de Gini. El índice de Gini, que proviene de la teoría de distribución de la renta y ha sido incorporado a los análisis de localización industrial, toma valores entre 0 y I, mide el grado de concentración de un mercado. Para valores del índice cercanos a cero la interpretación es que existe una baja o nula concentración en el mercado de análisis, mientras que para índices cercanos a uno la interpretación es que existe una muy alta concentración de mercado.

2 Los KIS_HT están compuesto por los CNAE 64, 72 y 73.

3 NUT (Nomenclature of Territorial Units) de Eurostat. La definición de las unidades territoriales se basa en las unidades administrativas existentes en los Estados miembros. Una unidad administrativa designa una zona geográfica con una autoridad administrativa que tenga poder para tomar decisiones de carácter administrativo o político dentro del marco jurídico e institucional del Estado miembro. En España el nivel NUT 2 corresponde a las Comunidades Autónomas.

4 Directorio Central de Empresas

5 http://epp.eurostat.ec.europa.eu/ 


\section{Análisis de variables y principales resultados}

\section{Número de empresas}

Comenzamos analizando la variable de número de empresas. De esta manera y según los datos del DIRCE había en España un total de 36.080 empresas KIS_HT en el año 2000 que han crecido hasta las 56.548 del año 2006. Según se puede observar en la Tabla 2, Madrid es la Comunidad que lidera el sector
KIS_HT por número de empresas en España,ya que en el 2006 esta comunidad acumulaba el $27,04 \%$ del total nacional de empresas, con un crecimiento de más de un punto porcentual desde el 25,97\% del año 2000. Cataluña se sitúa en segunda posición con el $23,16 \%$ de las empresas KIS_HT, perdiendo peso a nivel nacional en esta variable. Las siguientes posiciones las ocupan las comunidades autónomas de Andalucía y Comunidad Valenciana que entre las dos superan el $20 \%$ de las empresas existentes a nivel nacional para el año 2006.

Tabla 2

Distribución del número de empresas por comunidades autónomas KIS_HT (2000-2006). Fuente: Elaboración propia a partir de datos de DIRCE.

\begin{tabular}{|c|c|c|}
\hline \multirow[t]{2}{*}{ Comunidad Autónoma } & \multicolumn{2}{|c|}{ Años } \\
\hline & 2000 & 2006 \\
\hline Andalucía & $10.50 \%$ & $11.29 \%$ \\
\hline Aragón & $2.05 \%$ & $2.28 \%$ \\
\hline Asturias (Principado de) & $1.83 \%$ & $1.56 \%$ \\
\hline Balears (Illes) & $2.41 \%$ & $2.52 \%$ \\
\hline Canarias & $3.35 \%$ & $3.54 \%$ \\
\hline Cantabria & $0.91 \%$ & $0.83 \%$ \\
\hline Castilla y León & $3.66 \%$ & $3.29 \%$ \\
\hline Castilla-La Mancha & $1.68 \%$ & $2.01 \%$ \\
\hline Cataluña & $25.57 \%$ & $23.16 \%$ \\
\hline Comunidad Valenciana & $9.69 \%$ & $9.46 \%$ \\
\hline Extremadura & $0.92 \%$ & $1.05 \%$ \\
\hline Galicia & $4.03 \%$ & $4.05 \%$ \\
\hline Madrid (Comunidad de) & $25.97 \%$ & $27.04 \%$ \\
\hline Murcia (Región de) & $2.12 \%$ & $2.27 \%$ \\
\hline Navarra (Comunidad Foral de) & $1.32 \%$ & $1.30 \%$ \\
\hline País Vasco & $3.28 \%$ & $3.67 \%$ \\
\hline La Rioja & $0.57 \%$ & $0.52 \%$ \\
\hline Total nacional & $100.0 \%$ & $100.0 \%$ \\
\hline
\end{tabular}


A la vista de los datos anteriores, podemos apuntar que el mercado KIS_HT aparece como un mercado concentrado en las CCAA de Madrid y Cataluña, atendiendo esta vez a la variable del número de empresas. Sin embargo, para determinar el nivel de concentración, calculamos los índices de Gini para el periodo 2000-2006 para el sector KIS_HT y cada uno de los subsectores que lo componen, datos que pueden verse en el gráfico I.

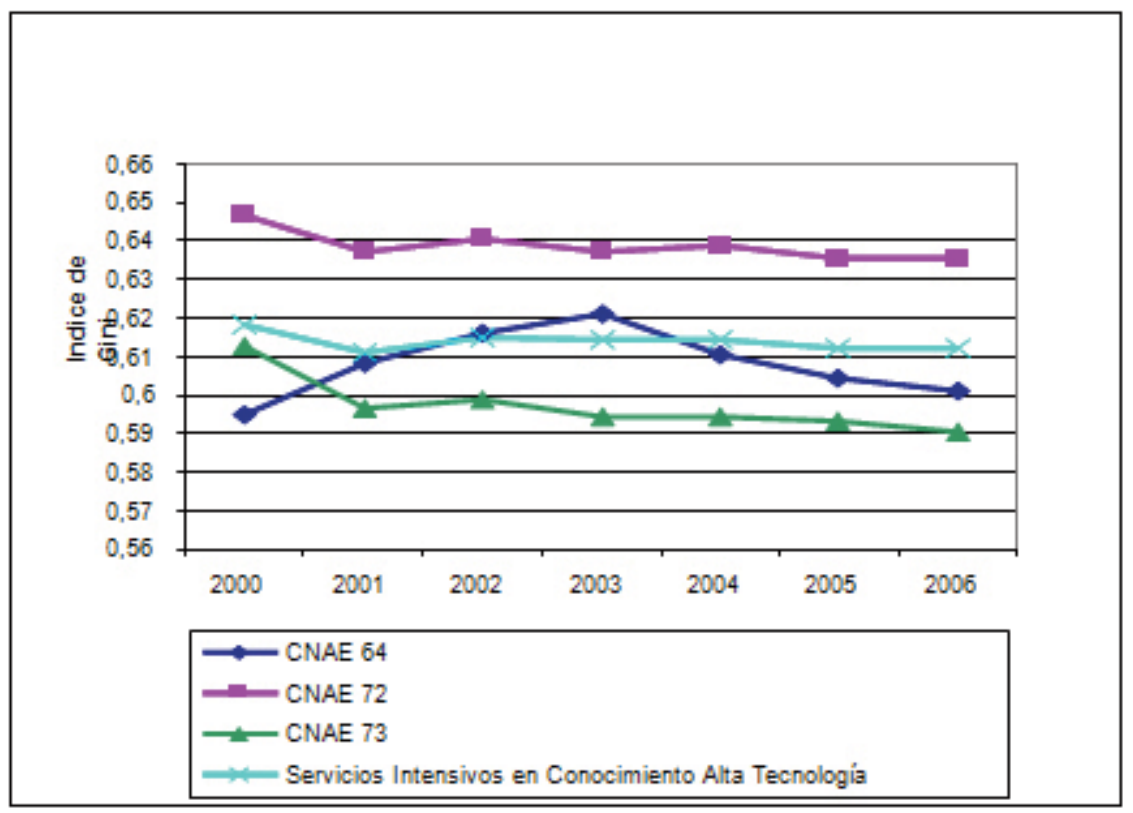

Gráfico I. Índice de concentración - Número de empresas KIS_HT en España (2000-2006). Fuente: Elaboración propia a partir de datos del DIRCE.

Lo primero que podemos añadir, a la vista de los resultados, es que el mercado KIS_HT en España, atendiendo a la variable de número de empresas, es un mercado concentrado, presentando para el año 2000 un índice de Gini de 0,62. La evolución que ha tenido la concentración del mercado KIS_HT ha sido estable con un valor del índice para 2006 cercano al 0,61. Tampoco se han producido variaciones relevantes en los años intermedios.

Atendiendo a los distintos subsectores, podemos decir que la evolución del CNAE 64 no ha presentado grandes oscilaciones, ya que, tras ser el sector menos concentrado de los tres considerados, la tendencia ha sido a un ligero aumento de la concentración en los primeros años, exactamente hasta el 0,62 en el año 2003, para volver a reducirse también ligeramente hasta el 0,6 en el año 2006. La tendencia de los CNAE 72 y 73 han sido también de estabilidad en la concentración. EI CNAE 72 era el subsector KIS_HT más concentrado. Este subsector presentaba índices cercanos a 0,65 para el año 2000, los cuales han permanecido estables hasta el 0,635 del año 2006.

Tal vez la conclusión más relevante del análisis de concentración referido al número de empresas sea por un lado la ausencia de variaciones importantes en el grado de concentración del sector KIS_HT en España,y por el otro, las diferencias en el grado de concentración existente entre los subsectores que lo componen, donde el sector de informática (CNAE 72) aparece como el sector más concentrado mientras que el sector de comunicaciones (CNAE 64) e investigación (CNAE 73) muestran un menor grado de concentración y sin grandes diferencias entre ellos.

Sin embargo, el análisis de la concentración atendiendo al número de empresas muestra lagunas importantes ya que la talla de la empresa no es tenida en cuenta en el mismo, pudiéndose dar la paradoja que un mayor número de empresas en una determinada zona geográfica no se tradujese en una mayor concentración en el resto de variables. Por ello y para explicar más convenientemente la concentración geográfica que se produce en el sector KIS_HT, vamos a ahondar en el análisis de concentración considerando la variable del número de ocupados.

\section{Número de ocupados}

La variable de número del ocupados parece resultar la más idónea para conducir el análisis ya que responde a los criterios de disponibilidad y representatividad. La disponibilidad hace referencia a la existencia de datos fiables provenientes de EUROSTAT y que con un nivel de desagregación de NUT 2 cubren periodos de análisis consistentes. Por su parte la representa- 
tividad hace referencia a la relevancia de la misma en relación con sectores que utilizan como primer factor productivo el capital humano. De la misma manera hay que apuntar que la variable de número de ocupados es la más utilizada en los análisis de concentración como medida del peso de un sector en un territorio determinado.

Según datos de EUROSTAT, España pasó de tener 209.982 ocupados (KIS_HT) en 1994 a 527.23I en 2006. Han sido las comunidades de Madrid, Cataluña, C.Valenciana, Andalucía y País

Tabla 3

Índices de especialización relativa en sectores KIS_HT. Fuente: Elaboración propia a partir de datos EUROSTAT.

\begin{tabular}{|c|c|c|c|c|c|c|c|c|c|c|c|c|c|}
\hline & 1994 & 1995 & 1996 & 1997 & 1998 & 1999 & 2000 & 2001 & 2002 & 2003 & 2004 & 2005 & 2006 \\
\hline España & 1.00 & 1.00 & 1.00 & 1.00 & 1.00 & 1.00 & 1.00 & 1.00 & 1.00 & 1.00 & 1.00 & 1.00 & 1.00 \\
\hline Galicia & 0.65 & 0.59 & 0.74 & 0.69 & 0.71 & 0.62 & 0.53 & 0.46 & 0.58 & 0.79 & 0.71 & 0.68 & 0.67 \\
\hline Principado de Asturias & 0.88 & 1.25 & 0.86 & 1.01 & 0.68 & 0.60 & 0.77 & 0.53 & 0.72 & 1.12 & 0.93 & 0.70 & 0.84 \\
\hline Cantabria & & & & & 0.83 & & & 0.48 & 0.68 & 0.76 & 0.67 & 0.48 & 0.81 \\
\hline País Vasco & 1.22 & 0.86 & 1.20 & 1.04 & 1.29 & 0.96 & 0.89 & 1.01 & 0.72 & 0.90 & 1.08 & 1.03 & 1.16 \\
\hline $\begin{array}{l}\text { Comunidad Foral } \\
\text { Navarra }\end{array}$ & & & & & & & 0.48 & 0.57 & 0.56 & 0.74 & 0.68 & 0.60 & 0.71 \\
\hline La Rioja & & & & & & & & & & & & & 0.66 \\
\hline Aragón & 0.86 & 0.88 & 0.86 & 0.73 & 0.66 & 0.61 & 0.52 & 0.64 & 0.68 & 0.98 & 0.91 & 0.85 & 0.94 \\
\hline Comunidad de Madrid & 2.12 & 2.34 & 2.37 & 2.06 & 2.35 & 2.46 & 2.59 & 2.75 & 2.63 & 2.18 & 2.30 & 2.20 & 2.09 \\
\hline Castilla y León & 0.80 & 0.70 & 0.73 & 0.75 & 0.70 & 0.79 & 0.78 & 0.60 & 0.71 & 0.79 & 0.62 & 0.63 & 0.69 \\
\hline Castilla-la Mancha & 0.85 & 0.67 & 0.48 & 0.54 & 0.42 & 0.44 & 0.58 & 0.54 & 0.48 & 0.58 & 0.40 & 0.52 & 0.59 \\
\hline Extremadura & 0.78 & 0.64 & 0.68 & 0.80 & 0.70 & 0.54 & 0.40 & 0.33 & 0.44 & 0.65 & 0.66 & 0.59 & 0.53 \\
\hline Cataluña & 1.01 & 1.02 & 0.96 & 1.14 & 1.16 & 1.04 & 1.10 & 1.06 & 1.09 & 1.06 & 1.04 & 1.07 & 1.03 \\
\hline Comunidad Valenciana & 0.78 & 0.80 & 0.77 & 0.86 & 0.56 & 0.89 & 0.68 & 0.70 & 0.62 & 0.60 & 0.65 & 0.72 & 0.88 \\
\hline Illes Balears & 0.70 & 0.72 & 0.62 & 0.78 & 0.58 & 0.98 & 0.78 & 0.76 & 0.62 & 0.60 & 0.61 & 0.52 & 0.44 \\
\hline Andalucía & 0.67 & 0.71 & 0.73 & 0.68 & 0.61 & 0.65 & 0.66 & 0.58 & 0.64 & 0.70 & 0.68 & 0.76 & 0.71 \\
\hline Región de Murcia & 0.67 & 0.46 & 0.47 & 0.51 & 0.78 & 0.44 & 0.45 & 0.45 & 0.51 & 0.73 & 0.65 & 0.45 & 0.51 \\
\hline Canarias & 0.92 & 0.65 & 0.69 & 0.86 & 0.71 & 0.50 & 0.62 & 0.57 & 0.52 & 0.66 & 0.66 & 0.86 & 0.72 \\
\hline
\end{tabular}


Vasco donde se ha concentrado este crecimiento. Esto lo podemos ver detalladamente analizando los índices de especialización relativa ${ }^{6}$ (1994-2006) para las distintas comunidades autónomas. En la tabla 3 podemos observar como Madrid es la comunidad con un índice de especialización mayor $(2,09)$, seguida a distancia por País Vasco y Cataluña. La comunidad menos especializada es Baleares.

Por lo que se refiere a la evolución en el periodo analizado no se ven demasiadas diferencias, siendo la tendencia hacia la estabilidad del peso relativo de este sector en las economías de las distintas CC.AA.Tal vez podemos resaltar las variaciones de especialización de la comunidad de Madrid, que en el año 200 I alcanzó un índice de 2,75 para caer hasta el 2,09 en el año 2006. Esta variación tienen su explicación en la pérdida de empleo debida a la crisis en el sector TIC que supuso la desaparición de hasta casi 30.000 empleos en España desde 200I hasta 2003, concentrándose principalmente esa pérdida en la Comunidad de Madrid.

Llama la atención también la pérdida de especialización relativa de comunidades como Islas Baleares, Extremadura, Murcia, Castilla la Mancha y en menor medida Castilla y León.

Por otro lado, calcularemos nuevamente el índice de Gini para la variable de número de ocupados. Podremos, por tanto, hacer una estimación del grado de concentración del mercado de servicios intensivos en conocimiento de alta tecnología (KIS_HT) mediante su aplicación. Los datos se pueden ver en el gráfico que se presenta a continuación.

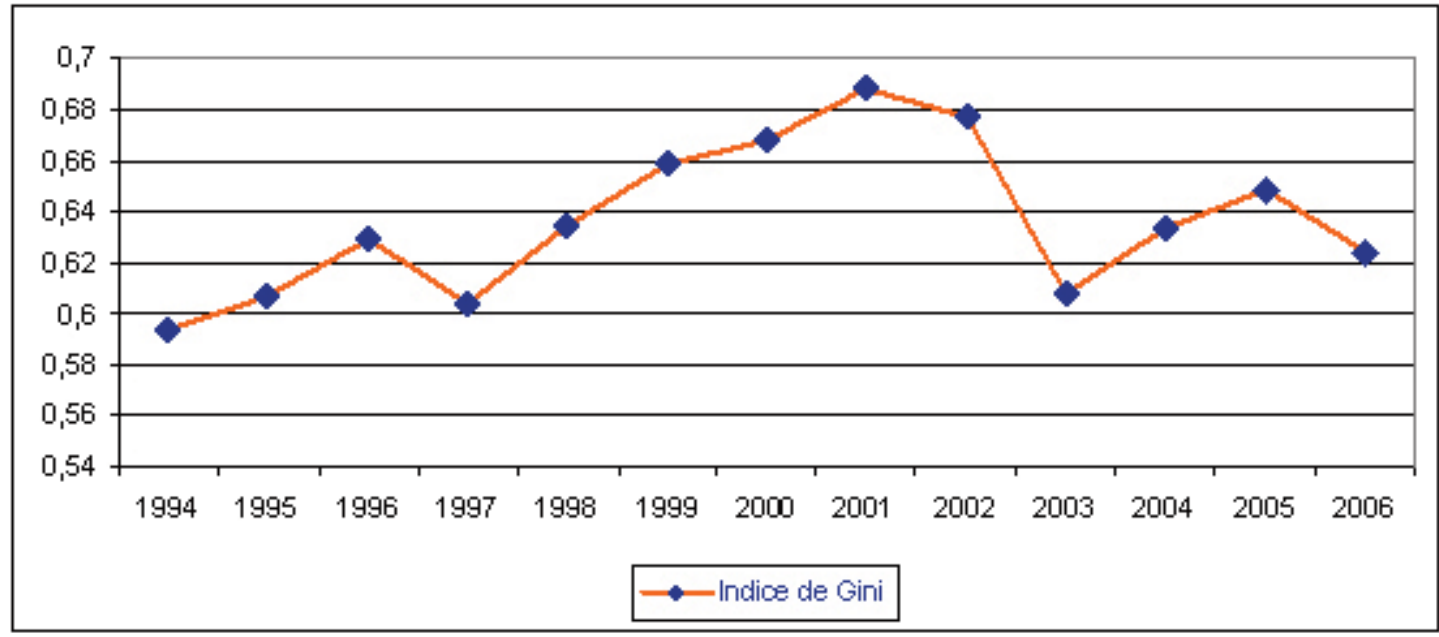

Gráfico 2. Índice de concentración de empleo KIS_HT sobre total de empleo (1994-2006). Fuente: Elaboración propia a partir de datos EUROSTAT

A la vista de los resultados podemos decir que aunque los sectores KIS_HT han sufrido variaciones en el grado de su concentración en el periodo analizado, estas han sido muy débiles y no han supuesto diferencias sustanciales respecto a las cifras del año 1994 y las de 2006. Sin embargo, sí que han existido variaciones en periodos intermedios donde los índices de concentración han sufrido altas y bajas notables, pudiendo ser explicado por los periodos de expansión y crisis en el sector de tecnologías de la información y telecomunicaciones que ha supuesto la acumulación y posterior pérdida de ocupación principalmente en la Comunidad de Madrid.
Es interesante ver la comparativa de concentración entre los años 2000 y 2006 atendiendo a las variables de número de ocupados y número de empresas. Como podemos observar en la Tabla 4, existen diferenciales de concentración entre las dos variables, existiendo significativa mayor concentración en términos de ocupados que de número de empresas desde los años 2000 hasta el 2003. Sin embargo el año 2003, año en el que la crisis en el sector TIC es más virulenta en España, las concentraciones atendiendo a las variables consideradas aparecen casi igualadas. Este hecho ha sido debido principalmente a la mayor destrucción de empleo que se ha dado en las comunidades de Madrid y Cataluña, lo que como ya hemos explicado descon-

6 Este índice se calcula midiendo el peso relativo que los ocupados en estos sectores tienen en una determinada región en función del peso medio que los ocupados en estos sectores tiene a nivel nacional. 
centró la ocupación KIS_HT en España.A partir del año 2003 se han seguido produciendo diferencias de concentración entre ambas variables pero más atenuadas con respecto al periodo anterior a la crisis.

\section{Tabla 4}

Diferenciales de concentración entre número de empresas y número de ocupados. Fuente:

\section{Elaboración propia.}

\begin{tabular}{llllllll}
\hline Gini & 2000 & 2001 & 2002 & 2003 & 2004 & 2005 & 2006 \\
\hline$N^{\circ}$ de ocupados & 0.6681 & 0.6878 & 0.677 & 0.6079 & 0.6332 & 0.6487 & 0.6241 \\
$\mathrm{~N}^{\circ}$ de empresas & 0.6181 & 0.6112 & 0.6151 & 0.6143 & 0.6146 & 0.6123 & 0.6123 \\
\hline Diferencial & 0.05 & 0.0766 & 0.0619 & -0.0064 & 0.0186 & 0.0364 & 0.0118 \\
\hline
\end{tabular}

\section{Salarios}

Si bien el número de ocupados es una variable que nos puede dar una medida fiable de la distribución y concentración del sector KIS_HT en España, es también interesante estudiar las variables que caracterizan ese empleo. En este sentido existen datos disponibles de los salarios del sector KIS_HT en España provenientes de EUROSTAT.

A la vista de los datos disponibles en el gráfico 2, podemos decir que el crecimiento de los salarios ha sido la tónica gen- eral en todos los subsectores $y$ en el sector KIS_HT en general. Unos, como el sector de investigación y desarrollo, han presentado un crecimiento más fuerte, mientras que otros, como el de comunicaciones, presentan un crecimiento mucho más moderado, sobre todo desde el año 2002. El comportamiento de los salarios del sector informática, si bien han crecido ininterrumpidamente durante el periodo de análisis, también muestra una curva de crecimiento más moderada que el producido en el sector de investigación y desarrollo.

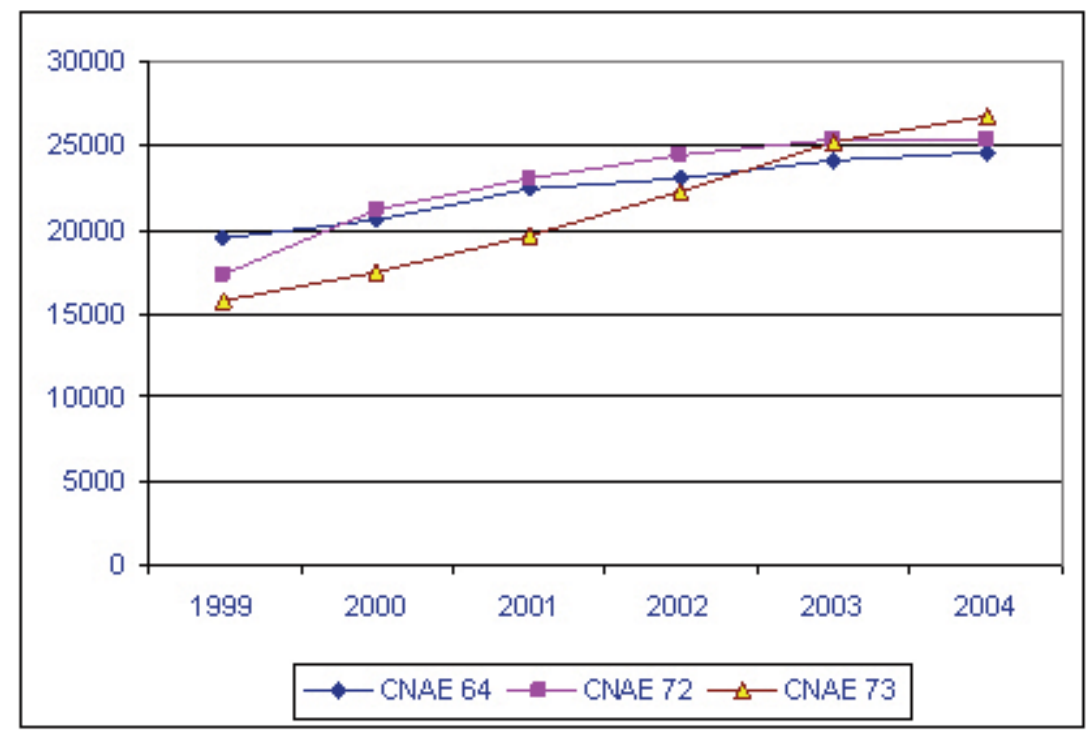

Gráfico 3. Evolución de los salarios medios en el sector servicios intensivos en conocimiento de alta tecnología en España (I999-2004). Fuente: Elaboración propia a partir de datos EUROSTAT. 
Para calcular la concentración atendiendo a la masa salarial, hemos calculado el índice de Gini para el año 2004. La falta de datos desglosado por comunidades autónomas para los años anteriores ha hecho inviable el cálculo de índices para los mismos. El resultado para el año 2004 ha sido de un índice de 0,89, lo que implica un grado de concentración muy alto atendiendo a este variable. Parece ser, que además de una concentración de la actividad de los servicios intensivos en conocimiento de alta tecnología en determinadas comunidades autónomas, también existe una alta concentración de las actividades de mayor valor añadido a nivel geográfico. Esta hipótesis puede ser refrendada a través de la comparación entre el nivel de concentración existente entre el número de ocupados y el de su masa salarial. Si comparamos el índice de concentración atendiendo a los salarios con el del número de empresas $(0,61)$ y número de ocupados $(0,63)$ para el mismo año, podemos observar unos diferenciales importantes.

La lectura que hacemos es sencilla, frente a los ya altos niveles de concentración que muestra la variable de ocupación en sec- tores intensivos en conocimiento de alta tecnología, la concentración de la masa salarial de estos sectores es sensiblemente más alta y además alcanzando cuotas de concentración muy cercanas a los valores superiores del índice. Por tanto hemos de entender que las diferencias entre los valores medios de los salarios entre unas comunidades autónomas y otras son importantes.

\section{Comparativa con otros sectores}

Para concluir el análisis de concentración del sector KIS_HT en España es interesante contraponer los datos de concentración en este tipo de sectores con los de otros que incorporan las variables de intensidad de conocimiento $y$ de tecnología. De esta manera podremos sacar conclusiones de cómo influyen estas variables en el grado de concentración. Por ello presentamos a continuación los índices de concentración para el sector de servicios intensivos en conocimiento, los servicios intensivos en conocimiento de alta tecnología y los sectores industriales de alta tecnología.

\section{Tabla 5}

Índices de concentración por distintos sectores. Fuente: Elaboración propia a partir de datos EUROSTAT

\begin{tabular}{|c|c|c|c|c|c|c|c|c|c|c|c|c|c|}
\hline Año & 94 & 95 & 96 & 97 & 98 & 99 & 00 & 01 & 02 & 03 & 04 & 05 & 06 \\
\hline $\begin{array}{l}\text { SERVICIOS } \\
\text { INTENSIVOS EN } \\
\text { CONOCIMIENTO }\end{array}$ & 0.51 & 0.52 & 0.5 & 0.51 & 0.52 & 0.53 & 0.54 & 0.53 & 0.53 & 0.52 & 0.53 & 0.53 & 0.53 \\
\hline $\begin{array}{l}\text { SERVICIOS } \\
\text { INTENSIVOS EN } \\
\text { CONOCIMIENTO } \\
\text { ALTA } \\
\text { TECNOLOGIA }\end{array}$ & 0.59 & 0.60 & 0.62 & 0.60 & 0.63 & 0.65 & 0.66 & 0.68 & 0.67 & 0.60 & 0.63 & 0.64 & 0.62 \\
\hline $\begin{array}{l}\text { SECTORES } \\
\text { INDUSTRIALES DE } \\
\text { ALTA } \\
\text { TECNOLOGIA }\end{array}$ & 0.82 & 0.76 & 0.75 & 0.77 & 0.75 & 0.74 & 0.77 & 0.80 & 0.78 & 0.76 & 0.74 & 0.73 & 0.73 \\
\hline
\end{tabular}

Los comentarios que podemos realizar teniendo en cuenta la Tabla 5 son los siguientes. Podemos observar como el sector servicios intensivos en conocimiento (KIS) no muestra tendencias de concentración dignas de mención en el periodo analizado.

Ahora bien, veamos qué ocurre cuando consideramos la vari- able de intensidad tecnológica conjuntamente con la intensidad de conocimiento. Este hecho se ve reflejado en el análisis de los sectores KIS_HT realizado anteriormente y que presentaba una evolución hacia la estabilidad en la concentración pero con variaciones más importantes durante el periodo analizado. En este sentido nos interesa comparar las diferencias en las tendencias de concentración que encontramos entre los sectores 
intensivos en conocimiento (KIS) y los sectores intensivos en conocimiento de alta tecnología (KIS_HT). Así, vemos como existe un diferencial de concentración entre los sectores KIS y KIS_HT de casi un $0, \mathrm{I}$ el cual se ha mantenido a lo largo del tiempo. Este diferencial ha llegado a ser de 0,15 puntos en el año 200l para disminuir progresivamente hasta el año 2006. Por otra parte, la evolución de la concentración en ambos sectores presenta diferencias, ya que mientras que el sector KIS muestra una evolución más lineal, el sector KIS_HT muestra oscilaciones más importantes, principalmente ligadas a la evolución del sector de las comunicaciones (CNAE 64).

Siguiendo con la variable de intensidad tecnológica, y pese a no formar parte del sector servicios, se ha analizado a efectos comparativos la concentración existente en los sectores industriales de alta tecnología 7 . Los sectores industriales de alta tecnología han mostrado una desconcentración desde el año 1994 hasta el 2006, pasando de un valor del índice de 0,82 hasta un 0,73 , lo que ha supuesto la pérdida de casi 0,1 puntos. Sin embargo estos sectores muestran un diferencial de concentración con los KIS_HT de 0,09 puntos en el año 2006, diferencial que ha disminuido ostensiblemente desde el año 1994 en el que se encontraba en 0,23 puntos.

\section{Conclusiones.}

A la vista de los resultados que han sido presentados, podemos aportar las siguientes conclusiones referentes al sector de servicios intensivos en conocimiento de alta tecnología.

Apuntamos que este sector en España muestra unos claros síntomas de concentración en determinadas comunidades autónomas, lo que se demuestra por la existencia de unos altos valores de los índices de concentración (Gini) analizados. Los valores de los índices presentan el mismo comportamiento para todas las variables de número de empresas y número de ocupados, no teniendo datos de evolución de la concentración para los salarios, aunque para el año analizado la concentración es la más alta de las variables analizadas.

La concentración de este sector se vincula a las comunidades autónomas de Madrid y de Cataluña, en consonancia con lo ya apuntado por autores como Mas (1992) que hacía referencia a la tendencia de concentración de este tipo de sectores en las grandes ciudades y en particular en las capitales.

Dentro de los tres subsectores que componen el sector de servicios intensivos en conocimiento de alta tecnología, es el sector de informática (CNAE 72) el que presenta un índice de concentración más alto, siempre por encima del sector de comunicaciones (CNAE 64) y del sector de investigación y desarrollo (CNAE 73).

La tendencia en la evolución de este tipo de sectores ha sido hacia el crecimiento, tanto en número de empresas, como en número de ocupados, como en el nivel salarial del sector y de cada uno de los subsectores. Por su parte, este crecimiento del sector no ha supuesto cambios significativos en las cifras finales del grado de concentración del mismo.

Sin embargo, dentro de los periodos de análisis, sí que ha habido fluctuaciones en el grado de concentración del sector KIS_HT, a tenor de los datos que podemos observar de la concentración de la ocupación del sector.Así, desde el año 1997 hasta el $200 \mathrm{I}$, la concentración crece en el sector de forma ininterrumpida desde índices del 0,6 hasta el 0,68, lo que es bastante significativo. A partir del año 200 l y hasta el año 2003, coincidiendo con la crisis del sector TIC en España, se produce una desconcentración del mercado en cuanto a la ocupación se refiere, alcanzando de nuevo índices del 0,6. La explicación de este comportamiento se atribuye a que tanto en épocas de expansión del sector como en épocas de crisis, el crecimiento de la ocupación o la destrucción de la misma está por encima de las medias de crecimiento en aquellos territorios más especializados sectorialmente, incrementando por tanto su especialización relativa. Así ha ocurrido en España, donde la comunidad de Madrid acaparó el crecimiento del sector entre el periodo 1997-200I, incrementando su índice de especialización relativa desde el 2,06 hasta el 2,75, para caer con posterioridad.

Sin embargo, las variaciones en la concentración de la ocupación no se encuentran en sintonía con la concentración del número de empresas, ya que este índice se mantiene en el 0,6I para el periodo analizado. Esto implica que la creación de empresas se realiza manteniendo la especialización relativa de cada territorio y que las comunidades que concentran el sector no crean proporcionalmente más número de empresas que aquellas que tienen una menor especialización.

En cuanto a la lectura que podemos hacer sobre los salarios, debemos tener en cuenta las restricciones del análisis de concentración sobre los mismos, ya que tan solo existen datos para el año 2004. Sin embargo, los diferenciales de concentración existentes entre número de ocupados y masa salarial implica que las comunidades autónomas que concentran la ocupación, concentran en mayor medida los salarios del sector. Ante este dato solo cabe una explicación y es que las actividades en las

7 Los KIS_HT junto con los sectores industriales de alta tecnología conforman el denominado sector de alta tecnología. 
que se especializa la actividad de las comunidades autónomas que lideran el sector en España sean las de mayor valor añadido y por lo tanto puedan de esta manera asumir unos salarios medios por encima de otras áreas.

Cabría analizar en futuras investigaciones la especialización de la producción por comunidades autónomas en función de los distintos subepígrafes. Es de esperar que las comunidades con menores salarios estén orientadas hacia actividades de menor valor añadido como pueden ser las actividades de apoyo a la investigación, la integración de programas informáticos, mantenimiento de equipos, las bases de datos o instalación de equipos de comunicaciones. Por otro lado, las comunidades autónomas con mayores salarios han de estar centradas en actividades de investigación y desarrollo puras, programas informáticos y desarrollo de software y aplicaciones y diseño y oferta de servicios de comunicaciones.

Por otra parte, la evolución de los índices de especialización de las distintas comunidades autónomas nos hace pensar en las dificultades de romper la inercia ya establecida y ganar especialización relativa en el sector.Vemos como son las comunidades líderes las que monopolizan las fases de crecimiento del sector incrementando su participación relativa en el mismo. De la misma manera, las fases de ajuste o crisis son también sufridas especialmente en estas comunidades.

Para concluir, hemos de hacer notar los diferenciales de concentración existente entre los sectores intensivos en conocimiento y los KIS_HT, donde parece que la intensidad tecnológica aporta un diferencial en el grado de concentración de estos sectores, diferencial que se hace más importante si consideramos los sectores industriales de alta tecnología. Estos datos van en consonancia con las aportaciones de autores como Vence (2007) que ya apuntaba que este tipo de servicios son los más concentrados, por encima de otro tipo de servicios intensivos en conocimiento.

\section{Referencias}

ABRAMOVITZ, M. y David, P. (1996). Technological change and the rise of intangible investments : the US Economy's growthpath in the twentieth century, en D. Foray y B.A. Lundvall (eds.). Employment and Growth in the Knowledge-based Economy, documentos de la OCDE, París : OCDE.

DAVID, P. y Foray, D. (2002): "Fundamentos económicos de la sociedad del conocimiento”. Comercio Exterior, 52, 6, 472-490.
ESTEBAN Barranco, Jesús (2006). Los servicios intensivos en conocimiento tecnológico (TKIBS): Un instrumento para la competitividad de las empresas y las regiones. COTEC, Madrid.

FUCHS, V. (1968). The service economy. Columbia University Press. Nueva York.

HAUKNES, J. ( 1999). Knowledge Intensive Services; What's their role?. OECDE Business and Industry Policy Forum. Realising the Potential of the Service Economy: Facilitating Growth, Innovation and Competition. OCDE. Paris

HENG, T. el al (2002). Mapping Singapore's Knowledge-Based Economy Economic Survey of Singapore, Third Quarter 2002

MARTINELLI, F y Cavola, L. (2002); The influence of regional demand and institutions on the role of KIS en Wood, P.(Ed) (200I); Consultancy and Innovation. Routledge, London (209-243)

MAS, F. (1992): Servicios a empresas y competitividad industrial. Economía Industrial, Septiembre-Octubre, pp. 63-67

MILES, I., et al (1995) Knowledge Intensive Business Services: Their Role as Users, Carriers and Sources of Innovation. EIMS Publication No. I5, Innovation Programme, DGXIII, Luxembourg.

MULLER E. and Zenker A. (200I), Business services as actors of knowledge transformation: the role of KIBS in regional and national innovation systems. Research Policy, Vol. 30, pp. I 50 I-I 5 I 6.

RUBALCABA Bermejo, L. et alt. (1998). Crecimiento y geografia de los servicios a empresas en el contexto de la nueva sociedad servindustrial: el caso de la Comunidad de Madrid. Comunidad de Madrid y Universidad de Alcalá

STRAMBACH, S. (200I). Innovation Process and the Role of Knowledge-Intensive Business Services, en Koschatzky, K., Kulicke, M., y Zenker, A. (eds), Innovation Networks-Concepts and Challenges in the European Perspective. Heidelberg, New York: Physica-Verlag, 53-68. 458-479, University of Aix-Marseille, France.

SUNDBO, J. (2002) Networked/mobile ICT as a basis for the development of knowledge service concepts, en Proceedings of: 7th International Research Seminar in Service Management" ,pp. 458-479, University of Aix-Marseille, France.

VENCE, X. (2007): La terciarización y metropolización de la economía mundial: una aproximación a partir de las tendencias en la Unión Europea, IX Reunión de Economía Mundial. Madrid. 
Abril 2007.

VENCE, X.y Rodil, O. (2003): La Concentración regional de la política de I+D de la Unión Europea: el principio de cohesión en entredicho, Revista de Estudios Regionales. 65, 43-73.

VERMEULEN, P. (200I) Organizing Product Innovation in Financial Services. Nijmegen University Press

\section{Acerca del Autor}

Juan Vicente García Manjón ocupa en la actualidad el cargo de Vicerrector de Espacio Europeo y Empleo en la Universidad Europea Miguel de Cervantes (Valladolid-España), donde ejerce su labor docente. Doctor en Tecnologías de la Información y las Telecomunicaciones, licenciado en Ciencias Económicas y Empresariales y con estudios de postgrado en Marketing y Gestión de la Innovación Tecnológica, cuenta con más de 17 años de experiencia profesional. Ha trabajado como evaluador para la Comisión Europea dentro del VI y VII Programa Marco de I+D y ha sido Vicepresidente del Centro Tecnológico para el Desarrollo de las Telecomunicaciones en Castilla y León. Su investigación se centra en la economía del conocimiento y la gestión de la innovación. 